\title{
The Effect of Experience, Coaching and Technical Post, on the Coping Strategies Solicited by the U15 Elites Affiliated to the Regional Centers of the Tunisian Football Federation (TFF)
}

\author{
Jamel Hajji ${ }^{1,2}$, Ali Elloumi ${ }^{3,4}$ \\ ${ }^{1}$ Department of Sciences Education, Faculty of Humanities at Tunis, University of Tunis, Tunis, Tunisia \\ ${ }^{2}$ Higher Institute of Sport and Physical Education Gafsa, Gafsa University, Gafsa, Tunisia \\ ${ }^{3}$ Department of Arts and Sciences Social, Sfax University, Sfax, Tunisia \\ ${ }^{4}$ Laboratoire Techniques et Enjeux du Corps, Université Paris Descartes, Paris 5, France \\ Email: Hajji5jamel@yahoo.fr
}

How to cite this paper: Hajji, J., \& Elloumi, A. (2017). The Effect of Experience, Coaching and Technical Post, on the Coping Strategies Solicited by the U15 Elites Affiliated to the Regional Centers of the Tunisian Football Federation (TFF). Advances in Physical Education, 7, 107-122. https://doi.org/10.4236/ape.2017.71010

Received: January 25, 2017

Accepted: February 25, 2017

Published: February 28, 2017

Copyright $\odot 2017$ by authors and Scientific Research Publishing Inc. This work is licensed under the Creative Commons Attribution International License (CC BY 4.0).

http://creativecommons.org/licenses/by/4.0/

\begin{abstract}
Today, the training center represents an unavoidable passage in the career of the high-level footballer. Training centers are among the mechanisms of $\mathrm{Na}$ tional Technical Direction, to develop Tunisian football, and to improve performance in youth categories. The objective of our study is to examine the coping strategies of the competition, solicited by the elites affiliated to the regional training centers of the Tunisian Football Federation (TFF) and evaluate the interaction effects of coaching, experience, and the technical position. 76 U15 football players regularly evaluated through high-stakes competitions are invited in one hour after the competition, to settle against the Arabic version of the inventory of coping strategies of the sporting competition. Data were collected and analyzed by SPSS IBM and AMOS version 21.0.0. The results suggest that mental distraction is the coping strategy most solicited by our participants, the MANOVA analysis, only disclosed the effect of the coaching factor on mental imagery, thought control, Effort expenditure and Relaxation, but regression analysis revealed no strong explanatory relationship. Overall, this study allowed us to deepen our knowledge about the coping strategies of the competition used in the preformation process in the different training centers of the TFF. In addition, the impact of experience, coaching and the specificity of the technical position, on the choice of these strategies in com-
\end{abstract}


petitive contexts.

\section{Keywords}

Coping Strategies, Training Center, Tunisian Football Federation (TFF)

\section{Introduction}

In order to demonstrate their personal competence, elite footballers will be evaluated several times, (Martinent and Decret, 2015). An adequate transition period between the ages of 13 and 15 allows the athlete to achieve high-level performance and to specialize before the advanced years (16 and over), (Faure and Suaud, 1999; Slimani, 2000; Demazière and Csakavary, 2002, Bertrand, 2008; Roderick, 2006). Young people in this way face a double uncertainty, their chance to lead to the end, in which the achievement of a career rests on a virtuosity acquired early; And the initiation in which the environment plays a fundamental role (Bertrand, 2015). Moreover, coping with the stresses of sporting competition is an essential factor of self-regulation to promote optimal levels of sporting success (Gaudreau, Nicholls, \& Levy, 2010).

The treatment of the threatening action is always done only in relation to the answers that the individual judges himself capable or not to bring. It is in this treatment that the strategies of coping or coping, defined by Lazarus and Folkman, come into play as "the constantly changing cognitive and behavioral efforts deployed by the individual to respond to specific internal and/or external demands, which are assessed as very strong and exceeding its adaptive resources". Coping is a dynamic process, which changes according to situations and how the individual treats them. The athlete adopts this mechanism to manage the stressful stresses of competition and maximize performance (Calmeiro et al., 2010, 2014, Doron \& Gaudreau, 2014, Doron \& Martinent, 2016).

Carton-Caron (2004) states that the modes of "coping" are based on internal and/or external factors, indicating great inter and intra-individual variability. Some studies show that coping changes through the different phases of the competition (Gaudreau, Lapierre, Blondin, 2001, Gaudreau and Blondin, 2004).

For some authors, the determinants of coping are provisional. For others, coping is determined by situational or transactional characteristics. The transactional approach of Lazarus and Folkman (1984) is based on the principle that the individual permanently assesses his relationship with the environment and his report on personal well-being. In the spirit of the Lazarus model, coping has two essential functions, direct action on the causes of the problem, or moderation of the emotional consequences of the stressful interaction. Mellalieu, Hanton and Fletcher (2006), indicate that the level of the athlete's experience influences the choice of coping strategies used. Some authors point out that young people use emotion-based strategies, while others point to the use of problem-oriented strategies. The same applies to the elderly (Callahan and Chabrol, 2013). 
Cosh and Tully (2015), Anshel and Si (2008), identified that the behavior of the trainer (coaching) was described as a key stressor. The study by Gearity \& Murray (2011), on coaching, showed that the athletes indicated that the style of coaching was associated with their adaptation and their sports motivation. Support coaching can play a positive role in providing direction in the process of achieving goals and in promoting the development of athletic and mental skills (Côté et al., 1999). In this sense, it can also be considered as a potential resource (Hobfoll, 2002), to make athletes more capable of solving problems and to cope with the stresses inherent in sports competitions (Ntoumanis, Biddle and Haddock, 1999). Effective coaching requires not only the establishment of a satisfactory relationship, but also the physical, technical, mental and tactical preparation of athletes (Hollembeak and Amorose, 2005).

\section{Materials and Methods}

\subsection{Population}

76 footballers U15 (average age 14.00, SD: 0.33) (see Table 1) affiliated to the regional training centers.

The regional technical advisor appointed by the Tunisian Football Federation (TFF) selects the best talents at the age of 13 to 14 in their region, after having evaluated these athletes during their participation in the official competitions in their clubs. Template, speed, technical capacity, tactical intelligence and integration; Are the most adopted criteria.

The selected footballers are then invited to the regional training centers supervised by the National Technical Direction (NTD) of the TFF; To be submitted to a final prospecting program, consisting of a physical, technical, and tactical testing package.

Finally, the 20 best talents are maintained and submitted to a training and evaluation program for 2 years to join the national selection.

Table 1. Frequency tables.

\begin{tabular}{ccccccc}
\hline & & Age (SD) & $\begin{array}{c}\text { Body } \\
\text { weight (SD) }\end{array}$ & Height (SD) & Effective & $\%$ \\
\hline \multirow{2}{*}{$\begin{array}{c}\text { Training } \\
\text { Center }\end{array}$} & Gafsa & $13.85(0.37)$ & $48.65(7.64)$ & $161.70(8.42)$ & 20 & 26.3 \\
& Gabès & $14.10(0.37)$ & $52.25(10.22)$ & $164.25(8.86)$ & 20 & 26.3 \\
& Le Kef & $14.12(0.37)$ & $50.71(6.41)$ & $164.82(7.43)$ & 17 & 22.4 \\
Level & Less than 2 years & $14.00(0.00)$ & $51.42(7.86)$ & $164.88(8.08)$ & 26 & 34.2 \\
Experience & More than 2 years & $14.00(0.40)$ & $50.30(8.11)$ & $163.38(7.97)$ & 50 & 65.8 \\
& Goalkeeper & $13.90(0.57)$ & $52.00(13.40)$ & $164.40(11.55)$ & 10 & 13.2 \\
$\begin{array}{c}\text { Technical } \\
\text { position }\end{array}$ & Defender & $14.00(0.00)$ & $50.64(8.23)$ & $164.45(7.94)$ & 22 & 28.9 \\
& Midfielders & $14.00(0.32)$ & $50.57(5.95)$ & $162.90(7.58)$ & 21 & 27.6 \\
& Attacker & $14.04(0.38)$ & $50.26(6.82)$ & $164.04(6.99)$ & 23 & 30.3 \\
\hline
\end{tabular}




\subsection{Measure}

We examined coping strategies through the Arabic version of the Inventory of Coping Strategies in Sports Competition (Hajji et al., 2016). The ISCCS (Gaudreau and Blondin, 2002) is a questionnaire of 39 items. When handling the questionnaire, participants had to position themselves against a Likert scale ranging from 1 (not at all used) to 5 (used very frequently). The questionnaire consists of 10 subscales: Mental imagery, Thought Control, Effort Expenditure, Seeking Support, Relaxation, Logical Analysis, Venting of Unpleasant Emotions, Disengagement, Social Withdrawal, Mental Distraction.

\section{Procedure}

After authorization to conduct our study, we contacted the heads of each training center and the parents of all athletes (under 15 years old); through letters of recommendation certified by the national technical direction (DTN) of the Tunisian Football Federation. The coaches are then invited to engage in the evaluation process.

All parents of participants have given their informed written consent. Prior to the data collection, the athletes who agreed to participate and their parents were given ample information about the study's purpose and procedure, and were informed that the results would be made available after completion of the survey, Study is completed.

During their sectoral groupings, in December 2015 and March 2016, athletes were invited to position themselves against the Arab version of the ISCCS, one to two hours after the competition.

The questionnaire was then preceded by clear and concise instructions, indicating information concerning age, gender, sports discipline, technical post held, and level of experience. The data was then analyzed by SPSS IBM and AMOS version 21.0.0.

\section{Data Analysis}

We evaluated the psychometric properties of the Arabic version of the ISCCS (Hajji et al., 2016), through the correlation for the ratio of the subscales, through the analysis of Alpha Cronbach for the internal consistency of the ISCCS, and Through an exploratory and confirmatory factor analysis processed with SPSS and AMOS 21.0.0, in order to validate the structure and arrangement of the ISCCS factors.

We use several indices of adequacy (Hoyle \& Panter, 1995; Kline, 2005) to evaluate the fit models to data collected such as the $\chi^{2}$ statistic that overcomes the abnormality data (Satorra and Bentler, 1994), the compared fit index CFI (Bentler, 1990) and TLI (Tucker-Lewis Index), and the Root Mean Square error of approximation RMSEA (Browne \& Cudeck, 1993).

To reveal the level of coping in general and in relation to the factors: coaching, level of experience and technical post of each athlete, we focused the mean scores and the standard deviations of each coping strategy. 
To support the interaction effect of factors, coaching, level of experience and technical post on coping strategies implored by our elites, we performed an MNOVA analysis. Finally, and to explain the effects of existing interactions, we performed a multiple regression analysis (input method).

\section{Results}

Psychometric properties of ISCCS

1) The report of the subscales

Significant positive correlations at $p<0.05$ were observed (see Table 2).

2) The internal consistency of the ISCCS

The Cronbach a coefficient of the different subscales ranges from 0.71 to 0.87 , while the overall Cronbach $\alpha$ coefficient of the scale is 0.77 (See Table 3 ).

3) Exploratory Factor Analysis

a) the 6-factor model: task-oriented coping: TOC

-Determining $=3.478 \mathrm{E}-005$.

$-\mathrm{KMO}$ index and Bartlett test: 0.696 (Meaning of Bartlett $<0.001$ ).

-The saturations of each item on the 6-factor model (See Table 4).

b) the 4-factor model: emotion-oriented coping: EOC

Table 2. Inter-subscale correlations of ISCCS.

\begin{tabular}{|c|c|c|c|c|c|c|c|c|c|c|}
\hline & & 1 & 2 & 3 & 4 & 5 & 6 & 7 & 8 & 9 \\
\hline \multirow{2}{*}{ Mental imagery } & $\mathrm{r}$ & 1 & & & & & & & & \\
\hline & $p$ & & & & & & & & & \\
\hline \multirow{2}{*}{ Thought control } & $\mathrm{r}$ & 0.022 & 1 & & & & & & & \\
\hline & $p$ & 0.850 & & & & & & & & \\
\hline \multirow{2}{*}{ Effort expenditure } & $\mathrm{r}$ & 0.087 & 0.047 & 1 & & & & & & \\
\hline & $p$ & 0.457 & 0.690 & & & & & & & \\
\hline \multirow{2}{*}{ Seeking support } & $\mathrm{r}$ & -0.095 & 0.147 & -0.122 & 1 & & & & & \\
\hline & $p$ & 0.414 & 0.207 & 0.294 & & & & & & \\
\hline \multirow{2}{*}{ Relaxation } & $\mathrm{r}$ & -0.126 & -0.007 & $-0.315^{* *}$ & $0.344^{* *}$ & 1 & & & & \\
\hline & $p$ & 0.277 & 0.950 & 0.006 & 0.002 & & & & & \\
\hline \multirow{2}{*}{ Logical analysis } & $\mathrm{r}$ & 0.168 & 0.164 & $0.381^{* *}$ & -0.003 & -0.096 & 1 & & & \\
\hline & $p$ & 0.147 & 0.157 & 0.001 & 0.980 & 0.408 & & & & \\
\hline \multirow{2}{*}{$\begin{array}{c}\text { Venting of } \\
\text { unpleasant emotions }\end{array}$} & $\mathrm{r}$ & $0.232^{*}$ & 0.081 & 0.071 & -0.020 & 0.062 & 0.223 & 1 & & \\
\hline & $p$ & 0.044 & 0.486 & 0.542 & 0.864 & 0.596 & 0.053 & & & \\
\hline \multirow{2}{*}{ Disengagement } & $\mathrm{r}$ & -0.152 & -0.087 & 0.102 & $0.280^{*}$ & 0.134 & 0.038 & -0.007 & 1 & \\
\hline & $p$ & 0.190 & 0.455 & 0.380 & 0.014 & 0.248 & 0.742 & 0.950 & & \\
\hline \multirow{2}{*}{ Social withdrawal } & $\mathrm{r}$ & $-0.272^{*}$ & 0.071 & -0.003 & $0.287^{*}$ & $0.306^{* *}$ & 0.129 & 0.113 & $0.339^{* *}$ & 1 \\
\hline & $p$ & 0.017 & 0.544 & 0.982 & 0.012 & 0.007 & 0.267 & 0.332 & 0.003 & \\
\hline \multirow{2}{*}{ Mental distraction } & $\mathrm{r}$ & -0.030 & 0.189 & -0.034 & 0.185 & $0.348^{* *}$ & 0.096 & $0.237^{*}$ & 0.011 & $0.254^{*}$ \\
\hline & $p$ & 0.795 & 0.102 & 0.772 & 0.109 & 0.002 & 0.411 & 0.040 & 0.926 & 0.027 \\
\hline
\end{tabular}

${ }^{*}$ The correlation is significant at 0.05 (bilateral). ${ }^{*}$ The correlation is significant at 0.01 (bilateral). 
Table 3. Alpha Cronbach coefficient of the ISCCS.

\begin{tabular}{ccc}
\hline ISCCS Subscales & Alpha Cronbach & N \\
\hline Mental imagery & 0.871 & 4 \\
Thought control & 0.842 & 4 \\
Effort expenditure & 0.729 & 3 \\
Seeking support & 0.709 & 4 \\
Relaxation & 0.711 & 4 \\
Logical analysis & 0.719 & 4 \\
Venting of unpleasant emotions & 0.845 & 4 \\
Disengagement & 0.830 & 4 \\
Social withdrawal & 0.726 & 4 \\
Mental distraction & 0.825 & 4 \\
The ISCCS scale & 0.772 & 39 \\
\hline
\end{tabular}

Table 4. Standardized solutions for factor loadings for the task oriented coping (TOC).

\begin{tabular}{|c|c|c|c|c|c|c|}
\hline & 1 & 2 & 3 & 4 & 5 & 6 \\
\hline Item 31 & 0.895 & & & & & \\
\hline Item 11 & 0.842 & & & & & \\
\hline Item 1 & 0.839 & & & & & \\
\hline Item 21 & 0.795 & & & & & \\
\hline Item 34 & & 0.855 & & & & \\
\hline Item 26 & & 0.809 & & & & \\
\hline Item 16 & & 0.806 & & & & \\
\hline Item 6 & & 0.772 & & & & \\
\hline Item 18 & & & 0.787 & & & \\
\hline Item 36 & & & 0.692 & & & \\
\hline Item 28 & & & 0.658 & & & \\
\hline Item 8 & & & 0.594 & & & \\
\hline Item 29 & & & & 0.781 & & \\
\hline Item 9 & & & & 0.744 & & \\
\hline Item 19 & & & & 0.721 & & \\
\hline Item 37 & & & & 0.570 & & \\
\hline Item 35 & & & & & 0.799 & \\
\hline Item 17 & & & & & 0.770 & \\
\hline Item 27 & & & & & 0.608 & -0.378 \\
\hline Item 7 & & & 0.453 & & 0.577 & \\
\hline Item 24 & & & & & & 0.796 \\
\hline Item 14 & & & & & & 0.761 \\
\hline Item 4 & & & & 0.400 & & 0.644 \\
\hline
\end{tabular}

-Determining $=0.001$.

-KMO index and Bartlett test: 0.714 (Meaning of Bartlett < 0.001). 
-The saturations of each item on the 4-factor model (See Table 5).

4) Confirmatory Factor Analysis

The 6-factor and 4-factor model adjustment indices are presented in Table 6.

\subsection{Level of Coping}

The average scores and standard deviations of each subscale are presented in Table 7 and Table 8.

Generally mental distraction, Social withdrawal and disengagement, are the coping strategies most requested by our participants.

Compared to the training center, we have distinguished that the coping strategies most used are, the control of thoughts among the elites of Gafsa, the Social withdrawal among the elites of Sidi Bouzid, the relaxation among the elites of Gabès and the mental distraction among the elites of El Kef.

Compared to the experience, we found that both groups, implore more the mental distraction.

Compared to the technical position, we have shown that the mental distraction is more demanded in the defenders, the midfielders and the attackers. While the goalkeepers demand more Social withdrawal.

Table 5. Standardized solutions for factor loadings for the emotion oriented coping (EOC).

\begin{tabular}{ccccc}
\hline & 1 & 2 & 3 & 4 \\
\hline Item 2 & 0.848 & & & \\
Item 32 & 0.819 & & & \\
Item 22 & 0.809 & & & \\
Item 12 & 0.788 & 0.878 & & \\
Item 30 & & 0.816 & & \\
Item 20 & & 0.804 & & \\
Item 10 & 0.700 & 0.833 & \\
Item 38 & & & 0.816 & \\
Item 39 & & & 0.776 & 0.782 \\
Item 15 & & & 0.739 & 0.776 \\
Item 25 & & & \\
Item 5 & & & 0.533 \\
Item 3 & & & \\
Item 23 & & & \\
Item 13 & & & \\
Item 33 & & & & \\
\hline
\end{tabular}

Table 6. Confirmatory factor analysis of the ISCCS measurement model.

\begin{tabular}{lcccccc}
\hline & $\mathrm{X}^{2}$ & $\mathrm{X}^{2} / \mathrm{df}$ & $p$ & CFI & TLI & RMSEA \\
\hline 6-factor model & 251.84 & 1.17 & 0.048 & 0.93 & 0.92 & 0.048 \\
4-factor model & 130.44 & 1.33 & 0.016 & 0.93 & 0.91 & 0.066 \\
\hline
\end{tabular}


Table 7. The level of coping strategies among the FFT-U15 elites.

\begin{tabular}{|c|c|c|c|c|c|c|c|c|c|c|}
\hline & $\begin{array}{l}\text { Mental } \\
\text { imagery }\end{array}$ & $\begin{array}{l}\text { Thought } \\
\text { control }\end{array}$ & $\begin{array}{c}\text { Effort } \\
\text { expenditure }\end{array}$ & $\begin{array}{l}\text { Seeking } \\
\text { support }\end{array}$ & Relaxation & $\begin{array}{c}\text { Logical } \\
\text { analysis }\end{array}$ & $\begin{array}{c}\text { Venting of } \\
\text { unpleasant } \\
\text { emotions }\end{array}$ & Disengagement & $\begin{array}{c}\text { Social } \\
\text { withdrawal }\end{array}$ & $\begin{array}{l}\text { Mental } \\
\text { distraction }\end{array}$ \\
\hline Mean (SD) & $10.25(3.69)$ & $14.89(3.44)$ & $9.12(3.66)$ & $14.51(3.34)$ & $14.38(3.70)$ & $10.95(3.98)$ & $10.95(3.98)$ & $15.03(3.41)$ & $15.17(3.54)$ & $\begin{array}{l}15.93 \\
(3.26)\end{array}$ \\
\hline $\mathrm{N}$ & & & & & & 76 & & & & \\
\hline
\end{tabular}

Table 8. The level of coping strategies in relation to factors: training center, experience and technical position.

\begin{tabular}{|c|c|c|c|c|c|c|c|c|c|c|c|}
\hline Training $\mathrm{C}$ & Center & $\begin{array}{l}\text { Mental } \\
\text { imagery }\end{array}$ & $\begin{array}{l}\text { Thought } \\
\text { control }\end{array}$ & $\begin{array}{c}\text { Effort } \\
\text { expenditure }\end{array}$ & $\begin{array}{l}\text { Seeking } \\
\text { support }\end{array}$ & Relaxation & $\begin{array}{l}\text { Logical } \\
\text { analysis }\end{array}$ & $\begin{array}{l}\text { Venting of } \\
\text { unpleasant } \\
\text { emotions }\end{array}$ & Disengagement & $\begin{array}{c}\text { Social } \\
\text { withdrawal }\end{array}$ & $\begin{array}{c}\text { Mental } \\
\text { distraction }\end{array}$ \\
\hline Gafsa & $\begin{array}{c}\text { Mean } \\
(\mathrm{SD})\end{array}$ & $\begin{array}{l}12.75 \\
(3.61)\end{array}$ & $\begin{array}{l}16.40 \\
(1.96)\end{array}$ & $\begin{array}{l}10.65 \\
(3.16)\end{array}$ & $\begin{array}{l}13.40 \\
(3.33)\end{array}$ & $\begin{array}{l}11.25 \\
(2.99)\end{array}$ & $\begin{array}{l}12.60 \\
(3.69)\end{array}$ & $\begin{array}{l}14.75 \\
(3.26)\end{array}$ & $\begin{array}{l}14.45 \\
(3.60)\end{array}$ & $\begin{array}{l}14.35 \\
(3.26)\end{array}$ & $\begin{array}{l}15.15 \\
(3.43)\end{array}$ \\
\hline S. Bouzid & $\begin{array}{c}\text { Mean } \\
(\mathrm{SD})\end{array}$ & $\begin{array}{l}08.32 \\
(2.52)\end{array}$ & $\begin{array}{l}14.31 \\
(4.62)\end{array}$ & $\begin{array}{l}09.84 \\
(3.71)\end{array}$ & $\begin{array}{l}14.74 \\
(3.62)\end{array}$ & $\begin{array}{l}15.26 \\
(2.96)\end{array}$ & $\begin{array}{l}12.00 \\
(4.72)\end{array}$ & $\begin{array}{l}14.79 \\
(4.32)\end{array}$ & $\begin{array}{l}15.52 \\
(3.39)\end{array}$ & $\begin{array}{l}16.84 \\
(3.08)\end{array}$ & $\begin{array}{l}15.84 \\
(2.52)\end{array}$ \\
\hline Gabès & $\begin{array}{c}\text { Mean } \\
(\mathrm{SD})\end{array}$ & $\begin{array}{l}10.60 \\
(3.67)\end{array}$ & $\begin{array}{l}13.85 \\
(3.06)\end{array}$ & $\begin{array}{l}06.10 \\
(2.81)\end{array}$ & $\begin{array}{l}15.85 \\
(2.87)\end{array}$ & $\begin{array}{l}17.15 \\
(2.47)\end{array}$ & $\begin{array}{l}08.85 \\
(3.33)\end{array}$ & $\begin{array}{l}15.50 \\
(2.98)\end{array}$ & $\begin{array}{l}15.45 \\
(3.75)\end{array}$ & $\begin{array}{l}16.05 \\
(3.62)\end{array}$ & $\begin{array}{l}16.85 \\
(1.90)\end{array}$ \\
\hline Le Kef & $\begin{array}{c}\text { Mean } \\
(\mathrm{SD})\end{array}$ & $\begin{array}{l}09.06 \\
(3.30)\end{array}$ & $\begin{array}{l}15.00 \\
(3.33)\end{array}$ & $\begin{array}{l}10.06 \\
(3.17)\end{array}$ & $\begin{array}{l}14.00 \\
(3.22)\end{array}$ & $\begin{array}{l}13.82 \\
(3.64)\end{array}$ & $\begin{array}{l}10.29 \\
(3.03)\end{array}$ & $\begin{array}{l}15.17 \\
(3.14)\end{array}$ & $\begin{array}{l}15.29 \\
(3.60)\end{array}$ & $\begin{array}{l}16.64 \\
(2.52)\end{array}$ & $\begin{array}{l}17.35 \\
(1.83)\end{array}$ \\
\hline \multicolumn{12}{|c|}{ Level of experience } \\
\hline $\begin{array}{l}\text { Less } 2 \\
\text { years }\end{array}$ & $\begin{array}{c}\text { Mean } \\
(\mathrm{SD})\end{array}$ & $\begin{array}{l}11.65 \\
(3.86)\end{array}$ & $\begin{array}{l}14.65 \\
(4.20)\end{array}$ & $\begin{array}{l}10.00 \\
(3.12)\end{array}$ & $\begin{array}{l}14.11 \\
(2.82)\end{array}$ & $\begin{array}{l}13.92 \\
(3.56)\end{array}$ & $\begin{array}{l}11.23 \\
(3.37)\end{array}$ & $\begin{array}{l}15.73 \\
(2.96)\end{array}$ & $\begin{array}{l}14.88 \\
(3.95)\end{array}$ & $\begin{array}{l}16.11 \\
(2.97)\end{array}$ & $\begin{array}{l}16.27 \\
(2.44)\end{array}$ \\
\hline $\begin{array}{c}\text { More } 2 \\
\text { years }\end{array}$ & $\begin{array}{c}\text { Mean } \\
(\mathrm{SD})\end{array}$ & $\begin{array}{l}09.52 \\
(3.41)\end{array}$ & $\begin{array}{l}15.02 \\
(3.00)\end{array}$ & $\begin{array}{l}08.66 \\
(3.82)\end{array}$ & $\begin{array}{l}14.72 \\
(3.58)\end{array}$ & $\begin{array}{l}14.62 \\
(3.77)\end{array}$ & $\begin{array}{l}10.80 \\
(4.29)\end{array}$ & $\begin{array}{l}14.70 \\
(3.60)\end{array}$ & $\begin{array}{l}15.32 \\
(3.34)\end{array}$ & $\begin{array}{l}15.84 \\
(3.43)\end{array}$ & $\begin{array}{l}16.26 \\
(2.73)\end{array}$ \\
\hline \multicolumn{12}{|c|}{ Technical post } \\
\hline Goalkeeper & $\begin{array}{c}\text { Mean } \\
(\mathrm{SD})\end{array}$ & $\begin{array}{l}09.80 \\
(3.42)\end{array}$ & $\begin{array}{l}15.30 \\
(4.44)\end{array}$ & $\begin{array}{l}08.30 \\
(3.88)\end{array}$ & $\begin{array}{l}14.10 \\
(3.90)\end{array}$ & $\begin{array}{l}15.40 \\
(4.29)\end{array}$ & $\begin{array}{l}08.50 \\
(4.88)\end{array}$ & $\begin{array}{l}13.40 \\
(3.89)\end{array}$ & $\begin{array}{l}15.00 \\
(5.45)\end{array}$ & $\begin{array}{l}16.50 \\
(3.03)\end{array}$ & $\begin{array}{l}15.90 \\
(2.84)\end{array}$ \\
\hline Midfielders & $\begin{array}{c}\text { Mean } \\
(\mathrm{SD})\end{array}$ & $\begin{array}{l}10.62 \\
(4.22)\end{array}$ & $\begin{array}{l}14.24 \\
(3.83)\end{array}$ & $\begin{array}{l}08.24 \\
(4.09)\end{array}$ & $\begin{array}{l}14.38 \\
(4.27)\end{array}$ & $\begin{array}{l}14.09 \\
(4.01)\end{array}$ & $\begin{array}{l}09.47 \\
(3.58)\end{array}$ & $\begin{array}{l}16.29 \\
(2.22)\end{array}$ & $\begin{array}{l}15.66 \\
(2.43)\end{array}$ & $\begin{array}{l}16.00 \\
(3.75)\end{array}$ & $\begin{array}{l}16.47 \\
(2.46)\end{array}$ \\
\hline Attacker & $\begin{array}{c}\text { Mean } \\
(\mathrm{SD})\end{array}$ & $\begin{array}{c}10.87 \\
(3.33)\end{array}$ & $\begin{array}{l}15.52 \\
(2.55)\end{array}$ & $\begin{array}{l}09.56 \\
(3.13)\end{array}$ & $\begin{array}{l}14.35 \\
(2.49)\end{array}$ & $\begin{array}{l}13.13 \\
(3.58)\end{array}$ & $\begin{array}{l}12.87 \\
(3.02)\end{array}$ & $\begin{array}{l}15.08 \\
(3.60)\end{array}$ & $\begin{array}{l}14.43 \\
(4.32)\end{array}$ & $\begin{array}{l}15.22 \\
(3.54)\end{array}$ & $\begin{array}{l}15.95 \\
(3.27)\end{array}$ \\
\hline
\end{tabular}

The interaction effect of coaching, experience and technical position.

The analysis of variance validated only the effect of the coaching center on the coping strategies of competition among our elites, [Wilks' Lambda $=0.233<1$, $\mathrm{D}=2.21, p=0.002<0.05$.] (See Table 9).

The strategies involved are mental imaging $(\mathrm{F}=2.882, p=0.046<0.05)$, thought control $(\mathrm{F}=3.17 ; p=0.036<0.05)$, Effort expenditure $(\mathrm{F}=3.969 ; p=$ $0.014<0.05)$ and relaxation $(\mathrm{F}=6.508 ; p=0.001<0.05)($ See Table 10$)$. 
Table 9. The effect of interaction between factors (IV) and strategies of coping (DV).

\begin{tabular}{ccccccc}
\hline & Effect & Value & D & $\begin{array}{c}\text { Ddl of the } \\
\text { hypothesis }\end{array}$ & ddl error & Sig. \\
\hline \multirow{2}{*}{$\begin{array}{c}\text { Training } \\
\text { Center } \\
\text { (coaching) }\end{array}$} & Trace of Pillai & 1.070 & 2.052 & 30.000 & 111.000 & 0.004 \\
& Wilks' Lambda & $\mathbf{0 . 2 3 3}$ & $\mathbf{2 . 2 1 6}$ & $\mathbf{3 0 . 0 0 0}$ & $\mathbf{1 0 3 . 4 0 8}$ & $\mathbf{0 . 0 0 2}$ \\
& Hotelling Trace & 2.132 & 2.392 & 30.000 & 101.000 & 0.001 \\
Rey's Biggest Root & 1.517 & $5.613^{\mathrm{c}}$ & 10.000 & 37.000 & 0.000 \\
experience & Trace of Pillai & 0.211 & $0.935^{\mathrm{b}}$ & 10.000 & 35.000 & 0.514 \\
& Wilks' Lambda & 0.789 & $0.935^{\mathrm{b}}$ & 10.000 & 35.000 & 0.514 \\
& Hotelling Trace & 0.267 & $0.935^{\mathrm{b}}$ & 10.000 & 35.000 & 0.514 \\
& Roy's Biggest Root & 0.267 & $0.935^{\mathrm{b}}$ & 10.000 & 35.000 & 0.514 \\
Technical & Trace of Pillai & 0.810 & 1.369 & 30.000 & 111.000 & 0.122 \\
& Wilks' Lambda & 0.366 & 1.409 & 30.000 & 103.408 & 0.105 \\
& Hotelling Trace & 1.274 & 1.430 & 30.000 & 101.000 & 0.096 \\
& Roy's Biggest Root & 0.654 & $2.421^{\mathrm{c}}$ & 10.000 & 37.000 & 0.025 \\
\hline
\end{tabular}

Table 10. Inter-subject interaction effects between training center factor and coping strategies).

\begin{tabular}{|c|c|c|c|c|c|c|}
\hline Source & Dependent variable & Sum of type III squares & $\mathrm{ddl}$ & Mean of squares & $\mathrm{F}$ & $p$-value. \\
\hline \multirow{10}{*}{$\begin{array}{l}\text { Training } \\
\text { Center } \\
\text { (coaching) }\end{array}$} & Mental imagery & 87.564 & 3 & 29.188 & 2.882 & 0.046 \\
\hline & Thought control & 77.250 & 3 & 25.750 & 3.117 & 0.036 \\
\hline & Effort expenditure & 121.532 & 3 & 40.511 & 3.969 & 0.014 \\
\hline & Seeking support & 82.723 & 3 & 27.574 & 2.143 & 0.108 \\
\hline & Relaxation & 207.474 & 3 & 69.158 & 6.508 & 0.001 \\
\hline & Logical analysis & 72.319 & 3 & 24.106 & 2.250 & 0.096 \\
\hline & $\begin{array}{l}\text { Venting of unpleasant } \\
\text { emotions }\end{array}$ & 21.018 & 3 & 7.006 & 0.580 & 0.631 \\
\hline & Disengagement & 27.433 & 3 & 9.144 & 0.930 & 0.434 \\
\hline & Social withdrawal & 29.877 & 3 & 9.959 & 0.832 & 0.483 \\
\hline & Mental distraction & 62.374 & 3 & 20.791 & 2.737 & 0.055 \\
\hline
\end{tabular}

\subsection{Multiple Linear Regression}

To explain the effect of interaction, a multiple regression analysis (entry method) was performed, we revealed four explanatory relationships that predict the following coping strategies (see Table 11):

The mental imagery $\left[\mathrm{R}^{2}=0.157 ; \mathrm{F}=4.483\right.$ at $\left.p=0.006\right]$, determined by training center (coaching) factors ( $\beta=-0.264, \mathrm{t}=-2.440$ at $p=0.017$ ).

The Relaxation $\left[\mathrm{R}^{2}=0.161 ; \mathrm{F}=4.622\right.$ at $\left.p=0.005\right]$, determined by the training center (coaching) factors $(\beta=0.298, \mathrm{t}=2.748$ at $p=0.008)$ and technical positions $(\beta=-0.230, \mathrm{t}=-2.124$ at $p=0.037)$.

The logical analysis $\left[\mathrm{R}^{2}=0.145 ; \mathrm{F}=4.070\right.$ at $\left.p=0.010\right]$, determined by the technical positions $(\beta=0.241, \mathrm{t}=2.207$ at $p=0.031)$. And the mental distraction $\left[\mathrm{R}^{2}=0.106 ; \mathrm{F}=2.831\right.$ at $\left.p=0.044\right]$, determined by the training center (coaching) factors $(\beta=0.325, \mathrm{t}=2.902$ at $p=0.005)$ (See Table 12). 
Table 11. Table summary of models.

\begin{tabular}{|c|c|c|c|c|c|c|c|c|c|c|}
\hline \multirow[b]{2}{*}{ Dependent variable } & \multirow[b]{2}{*}{$\mathrm{R}$} & \multirow[b]{2}{*}{$\mathrm{R}^{2}$} & \multirow[b]{2}{*}{$\begin{array}{c}\mathrm{R}^{2} \\
\text { Adjusted }\end{array}$} & \multirow[b]{2}{*}{$\begin{array}{l}\text { Standard Error } \\
\text { of Estimate }\end{array}$} & \multicolumn{5}{|c|}{ Change in statistics } & \multirow[b]{2}{*}{$\begin{array}{l}\text { Durbin } \\
\text { Watson }\end{array}$} \\
\hline & & & & & $\begin{array}{c}\text { Variation } \\
\text { of } \mathrm{R}^{2}\end{array}$ & $\begin{array}{c}\text { Variation } \\
\text { of } F\end{array}$ & ddl1 & ddl2 & $\begin{array}{l}\text { Sig. Variation } \\
\text { of } \mathrm{F}\end{array}$ & \\
\hline Mental Imagery & $0.397^{\mathrm{a}}$ & 0.157 & 0.122 & 3.45881 & 0.157 & 4.483 & 3 & 72 & 0.006 & 1.657 \\
\hline Relaxation & $0.402^{\mathrm{a}}$ & 0.161 & 0.127 & 3.45661 & 0.161 & 4.622 & 3 & 72 & 0.005 & 1.389 \\
\hline Logical Analysis & $0.381^{\mathrm{a}}$ & 0.145 & 0.109 & 3.75882 & 0.145 & 4.070 & 3 & 72 & 0.010 & 1.429 \\
\hline Mental Distraction & $0.325^{\mathrm{a}}$ & 0.106 & 0.068 & 2.53370 & 0.106 & 2.831 & 3 & 72 & 0.044 & 1.696 \\
\hline
\end{tabular}

Table 12. Coefficients table.

\begin{tabular}{|c|c|c|c|c|c|c|c|c|c|c|c|}
\hline \multirow{2}{*}{\multicolumn{2}{|c|}{ Dependent variable }} & \multicolumn{2}{|c|}{$\begin{array}{l}\text { Non-standardized } \\
\text { coefficients }\end{array}$} & \multirow{2}{*}{$\begin{array}{c}\begin{array}{c}\text { Standardized } \\
\text { coefficients }\end{array} \\
\text { Beta }\end{array}$} & \multirow{2}{*}{$\mathrm{t}$} & \multirow{2}{*}{ Sig. } & \multicolumn{3}{|c|}{ Correlations } & \multicolumn{2}{|c|}{$\begin{array}{l}\text { Statistics of } \\
\text { collinearity }\end{array}$} \\
\hline & & A & $\begin{array}{c}\text { Standard } \\
\text { Error }\end{array}$ & & & & $\begin{array}{c}\text { Simple } \\
\text { Correlation }\end{array}$ & Partial & Part & Tolerance & VIF \\
\hline \multirow{3}{*}{$\begin{array}{l}\text { Mental } \\
\text { Imagery }\end{array}$} & (Constant) & 14.520 & 2.023 & & 7.177 & 0.000 & & & & & \\
\hline & coaching & -0.830 & 0.361 & -0.250 & -2.302 & 0.024 & -0.271 & -0.262 & -0.249 & 0.991 & 1.009 \\
\hline & Expérience & -2.042 & 0.837 & -0.264 & -2.440 & 0.017 & -0.276 & -0.276 & -0.264 & 0.998 & 1.002 \\
\hline \multirow{3}{*}{ Relaxation } & (Constant) & 13.261 & 2.022 & & 6.559 & 0.000 & & & & & \\
\hline & coaching & 0.991 & 0.360 & 0.298 & 2.748 & 0.008 & 0.321 & 0.308 & 0.297 & 0.991 & 1.009 \\
\hline & $\begin{array}{l}\text { Technical } \\
\text { positions }\end{array}$ & -0.823 & 0.387 & -0.230 & -2.124 & 0.037 & -0.257 & -0.243 & -0.229 & 0.992 & 1.008 \\
\hline \multirow{2}{*}{$\begin{array}{l}\text { Logical } \\
\text { Analysis }\end{array}$} & (Constant) & 11.274 & 2.199 & & 5.128 & 0.000 & & & & & \\
\hline & $\begin{array}{l}\text { Technical } \\
\text { positions }\end{array}$ & 0.929 & 0.421 & 0.241 & 2.207 & 0.031 & 0.265 & 0.252 & 0.240 & 0.992 & 1.008 \\
\hline Mental & (Constant) & 14.533 & 1.482 & & 9.806 & 0.000 & & & & & \\
\hline Distraction & coaching & 0.767 & 0.264 & 0.325 & 2.902 & 0.005 & 0.324 & 0.324 & 0.323 & 0.991 & 1.009 \\
\hline
\end{tabular}

\section{Discussion}

\subsection{Psychometric Properties}

For Relationships between the ISCCS subscales, Correlation values range from 0.23 to 0.38 , so they are within an acceptable range (Briggs and Cheeks, 1986), (see Table 2).

For the internal consistency of the scale, the coefficients of a Cronbach are acceptable and similar to that of the original version of (Gaudreau \& Blondin, 2002) and the Arab version of ISCCS (Hajji et al., 2016). In general, according to De Vellis (1991), alpha values greater than 0.60 are considered acceptable. (See Table 3).

For confirmatory factor analysis revealed a good fit for both models. The 6-factor model [Chi-2 $=251.84$ at $p=0.048$; CFI and TLI are $>0.9$ and RMSEA $<$ 0.08 ], and the 4 -factor model [Chi-2 $=130.44$ at $p=0.016$; CFI and TLI are $>0.9$ and RMSEA < 0.08]. Tabachnick and Fidell (2007), (See Table 6 and Figure 1 and Figure 2). 


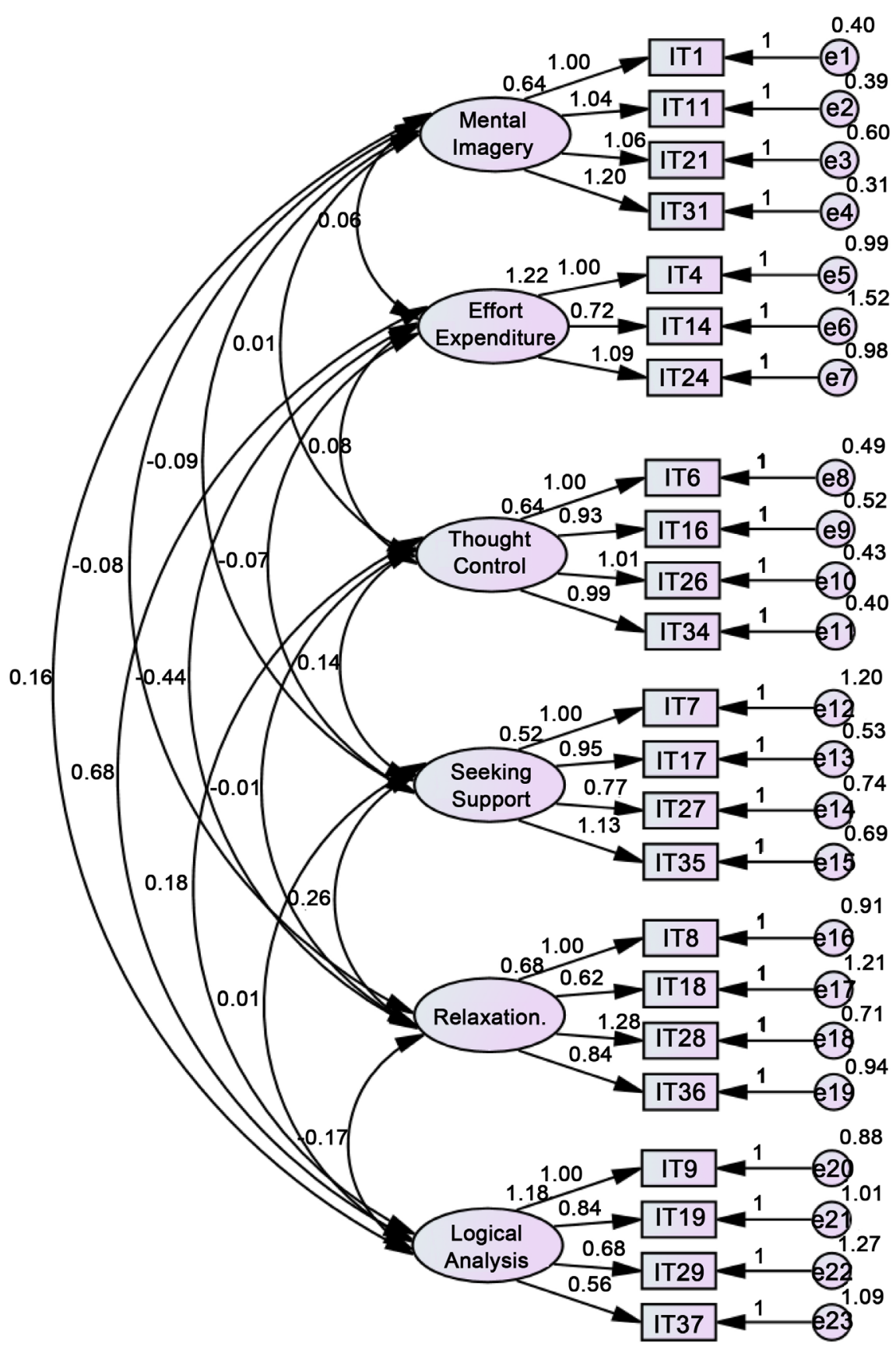

Figure 1. 6-factor model.

\subsection{Level of Coping and Anxiety}

The results of our study suggest that coping strategies oriented towards emotion, in a general way or in relation to factors, coaching, experience and technical positions, are most implored by our elites through the competitive environment. While some authors confirm (Gaudreau et al., 2002; Nicholls et al., Doron \& Gaudreau, 2014, Doron and Martinent, 2016) that task-oriented coping strategies are positively associated with performance, At the level of achievement the objectives of the competition (Amiot, Gaudreau and Blanchard, 2004; Dinca \& Rosnet, 2007). 


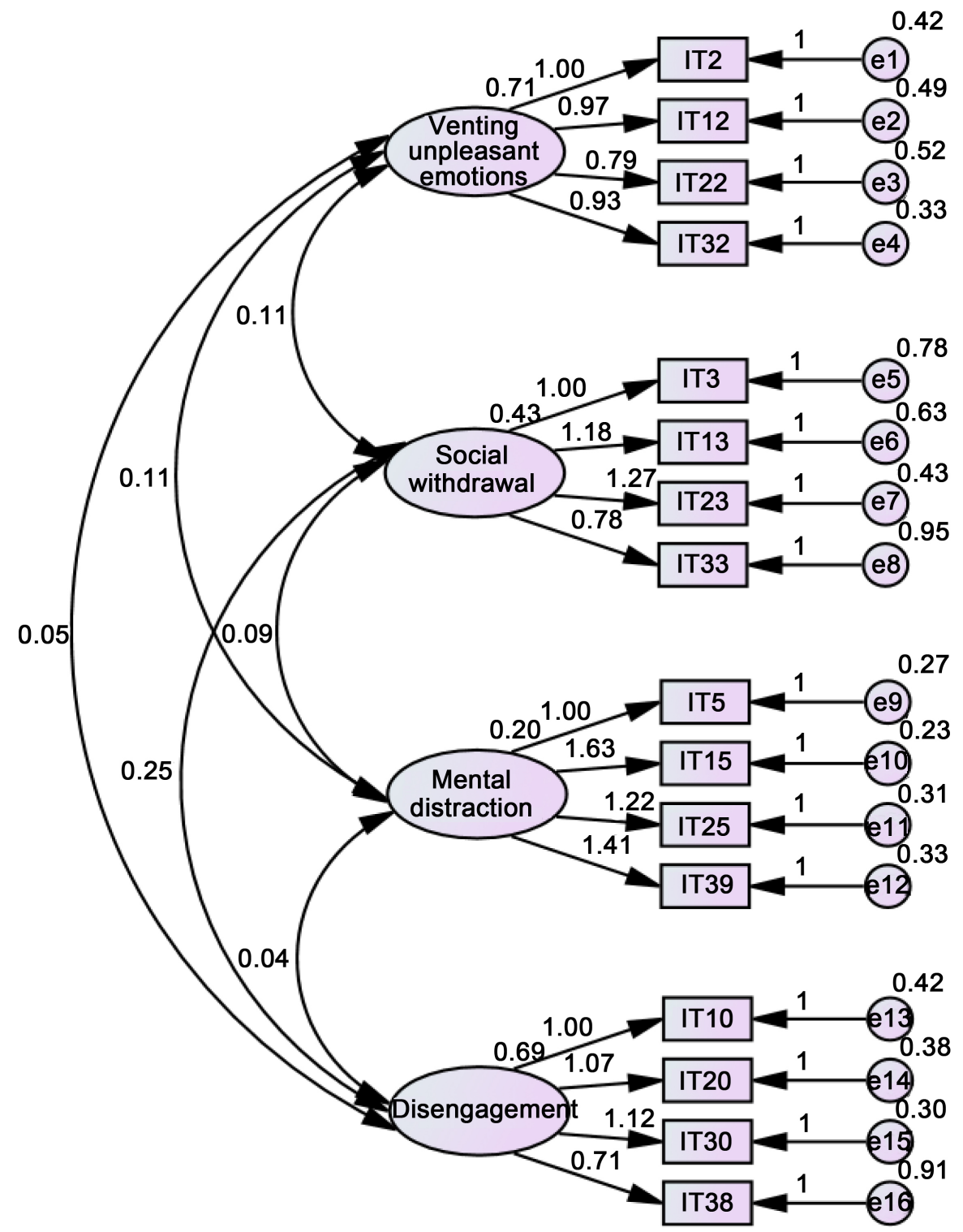

Figure 2. 4-factor model.

\subsection{The Interaction Effect of Determinants}

To estimate the interaction effect of the factors, the MANOVA variance analysis proves that only the training center or coaching factor has an interaction effect on task-oriented coping strategies such as imaging Mental control, thought control, effort deployment and relaxation. While the experience and the game post, have no interaction effect. Our results are similar to those published in the study by Nicolas, Gaudreau, and Franche (2011), who asserts that task-oriented coping strategies is an important process by which the Perceived support coaching has an influence on athletes during a specific competition. Similarly for Kristiansen et al. (2008), in elite athletes in four different European countries.

\subsection{Regression}

In order to know what factors (coaching, experience, technical position) influ- 
ence the coping strategies of the competition. We performed a multiple regression analysis, the input method.

The results suggest that:

The VIF and tolerance values confirm the absence of multicollinearity problem (see Table 12).

The Durbi-Watson test values for assessing the correlation between residuals and errors are in the range of 1.5 to 2.5 . The regression model is validated.

The coefficients of $\mathrm{F}$ obtained are significant at $p<0.05$, indicating that the model contribute to better predict coping strategies (Hair et al., 2010).

The results also suggest that there are only four significant explanatory relationships at $p<0.05$ ); Whereas the values of $\mathrm{R}^{2}$ indicate that the strength of all these relationships is very low (see Table 11).

$-15.7 \%$ of mental imagery is explained by factors, training center (coaching) and experience.

$-16.1 \%$ of relaxation is explained by the factors, training center (coaching) and Technical positions

$-14.5 \%$ logical analysis is explained only by the factor, gaming station

$-10.6 \%$ of the mental distraction is explained only by the factor, training center

The values of $\mathrm{R}^{2}$ indicate that the strength of all explanatory relationships is very low.

\section{Conclusion}

Research on coping in sports was strongly influenced by the transactional coping approach of Lazarus and Folkman (Nicholls \& Polman, 2007). In this context, our work was designed to provide a descriptive basis for understanding how our elites in regional state training centers manipulate coping strategies in key competitive events.

In the sports field, the use of task-oriented coping strategies and disengagement during a sporting competition are associated, positively and negatively, with the gap between the objectives set beforehand and the result achieved (Gaudreau \& Blondin, 2004; Gaudreau, Blondin, \& Lapierre, 2002), (Ntoumanis \& Biddle, 1998, Kim and Duda, 2003). Athletes who have a high use of task-oriented coping strategies, adapt better to the competition situation (Gaudreau \& Blondin, 2004). Contrary to what was expected, our elites are more impatient of emotion-based coping strategies.

Task-oriented coping strategies are associated with a more efficient organization of learning and working methods (Devonport \& Lane, 2006). Coaching is the effective determinants of training in the field of sport. In our work, analysis of variance revealed only the effect of training center where the trainer plays the crucial role, on the coping strategies used by our elites. Coaching has been established as a stressor in elite athletes. The flexibility and support of coaches was a crucial source for overcoming stressors (Cosh and Tully, 2015).

Today, applied research is needed to examine whether preventive psycho- 
educational interventions that teach coaching support behaviors (Smith, Smoll and Cumming, 2007) have effects on the use of athletes coping strategies.

\section{Limits}

Among the limitations of our work, only situational coping strategies have been examined, while coping strategies are also provisional, changing over time, situations and contexts (Gaudreau \& Miranda, 2010).

The second limit in our work is that there was no possibility to evaluate the coping during the competition (Gaudreau and Blondin, 2002).

\section{References}

Amiot, C. E., Gaudreau, P., \& Blanchard, C. M. (2004). Self-Determination, Coping, and Goal Attainment in Sport. Journal of Sport and Exercise Psychology, 26, 396-411. https://doi.org/10.1123/jsep.26.3.396

Anshel, M. H., \& Si, G. (2008). Coping Styles Following Acute Stress in Sport among Elite Chinese Athletes: A Test of Trait and Transactional Coping Theories. Journal of Sport Behavior, 31, 3-21.

Bentler, P. M. (1990). Comparative Fit Indexes in Structural Models. Psychological Bulletin, 107, 238-246. https://doi.org/10.1037/0033-2909.107.2.238

Bertrand, J. (2008). Preparing for the Profession of Footballer: Analysis of Professional Socialization. Staps, 82, 29-42. https://doi.org/10.3917/sta.082.0029

Bertrand, J. (2015). How Does One Become a Professional Footballer? Human Sciences, Monthly No. 272.

Briggs, S. R., \& Cheeks, J. M. (1986). The Role of Factor Analysis in the Development and Evaluation of Peronality Scales. Journal of Personality, 54, 106-148. https://doi.org/10.1111/j.1467-6494.1986.tb00391.x

Browne, V., \& Cudeck, R. (1993). Alternative Ways of Assessing Model Fit. In K. A. Bollen, \& J. S. Long (Eds.), Testing Structural Equation Models (pp. 136-162). Newbury Park, CA: Sage.

Callahan, S., \& Chabrol, H. (2013). Mechanisms of Defense and Coping (2nd ed.). Paris: Dunod.

Calmeiro, L., Tenenbaum, G., \& Eccles, D. (2010). Sequence Analysis of Appraisals and Coping during Trapshooting Performance. Journal of Applied Sport Psychology, 22, 392-407. https://doi.org/10.1080/10413200.2010.495325

Calmeiro, L., Tenenbaum, G., \& Eccles, D. (2014). Managing Pressure: Patterns of Appraisals and Coping Strategies of Non-Elite and Elite Athletes during Competition. Journal of Sports Sciences, 32, 1813-1820:

Carton-Caron, A. (2004). Coping Strategies in Athletes. Doctoral Thesis in Psychology, Bois: University of Charles de Gaulle Lille 3.

Cosh, S., \& Tully, J.P. (2015). Stresseurs d'adaptation et de mécanismes desoutien pour les étudiants-athlètes Combinant Elite Sport et enseignement supérieur: Implications pour lapratique. Texte intégral de l'article, Sport Psychologue.

Côté, J., Yardley, J., Hay, J., Sedgwick, W., \& Baker, J. (1999). An Exploratory Examination of the Coaching Behavior Scale for Sport. Avante, 5, 82-92.

Demazière, D., \& Csakavary, B. (2002). Becoming Professional. Panoramiques, 61, 85-91.

De Vellis, R. F. (1991) Scale Development: Theory and Application. London: Sage.

Devonport, T. J., \& Lane, A. M. (2006). Cognitive Appraisal of Dissertation Stress among 
Undergraduate Students. The Psychological Record, 56, 259-266.

Dinca, A., \& Rosnet, E. (2007). Relationship between Personal Effectiveness, Coping Strategies and Performance in Sports Competitions.

Doron, J., \& Gaudreau, P. A. (2014). Point by Point Analysis of Performance in a Fencing Match: Psychological Processes Associated with Winning and Losing Streaks. Journal of Sport and Exercise Psychology, 36, 3-13. https://doi.org/10.1123/jsep.2013-0043

Doron, J., \& Martinent, G. (2016). Trajectories of Psychological States of Women Elite Fencers during the Final Stages of International Matches. Journal of Sports Sciences, 34, 836-842. https://doi.org/10.1080/02640414.2015.1075056

Faure, J., \& Suaud, C. (1999). French Professional Football. Paris: University Press of France.

Gaudreau, P., \& Blondin, J. P. (2004). Different Athletes Cope Differently during a Sport Competition: A Cluster Analysis of Coping. Personality and Individual Differences, 36, 1865-1877. https://doi.org/10.1016/j.paid.2003.08.017

Gaudreau, P., \& et Blondin, J. (2002). Development of a Questionnaire for the Assessment of Coping Strategies Employed by Athletes in Competitive Sport Settings. Psychology of Sport and Exercise, 3, 1-34. https://doi.org/10.1016/S1469-0292(01)00017-6

Gaudreau, P., \& Miranda, D. (2010). Coping across Time, Situations, and Contexts: A Conceptual and Methodological Overview of Stability, Consistency, and Change. In A. R. Nicholls (Ed.), Coping in Sport: Concepts, Theories, and Related Constructs (pp. 15-32). New York: Nova.

Gaudreau, P., Lapierre, A. M., \& Blondin, J. P. (2001) Coping at Three Phases of a Competition: Comparison between Pre-Competitive, Competitive, and Post-Competitive Utilization of the Same Strategy. International Journal of Sport Psychology, 32, 369385.

Gaudreau, P., Nicholls, A., \& Levy, A. R. (2010). The Ups and Downs of Coping and Sport Achievement: An Episodic Process of Analysis within Person Associations. Journal of Sport \& Exercise Psychology, 32, 298-311. https://doi.org/10.1123/jsep.32.3.298

Gearity, B., \& Murray, M. (2011). Athletes' Experiences of the Psychological Effects of Poor Coaching. Psychology of Sport and Exercise, 12, 213-221. https://doi.org/10.1016/j.psychsport.2010.11.004

Hair, J. F., Black, W. C., Babin, B. J., \& Anderson, R. E. (2010). Multivariate Data Analysis (7th ed.). Upper Saddle River, NJ: Prentice Hall.

Hajji, J. (2016). Validation of the Arabic Version of the Inventory of Coping Strategies of Competitive Sport (ISCCS). Advances in Physical Education, 6, 312-327. https://doi.org/10.4236/ape.2016.64032

Hobfoll, S. E. (2002). Social and Psychological Resources and Adaptation. Review of General Psychology, 6, 307-324. https://doi.org/10.1037/1089-2680.6.4.307

Hollembeak, J., \& Amorose, A, J. (2005). Perceived Coaching Behaviors and College Athletes' Intrinsic Motivation: A Test of Self-Determination Theory. Journal of Applied Sport Psychology, 17, 20-36. https://doi.org/10.1080/10413200590907540

Hoyle, R. H., \& Panther, A. T. (1995). Writing about Structural Equation Models. In R. H. Hoyle (Ed.), Structural Equation Modeling: Concepts, Issues, and Applications (pp. 158-175). London: Sage.

Kim, M. S., \& Duda, J. (2003). The Coping Process: Cognitive Appraisals of Stress, Coping Strategies, and Coping Effectiveness. The Sport Psychologist, 17, 406-425. https://doi.org/10.1123/tsp.17.4.406

Kline, R. (2005). Principles and Practice of Structural Equation Modeling (2nd ed.). New York: Guilford. 
Kristiansen, E., Roberts, G. C., \& Abrahamsen, F. E. (2008). Achievement Involvement and Stress Coping in Elite Wrestling. Scandinavian Journal of Medicine \& Science in Sports, 18, 526-538. https://doi.org/10.1111/j.1600-0838.2007.00646.x

Lazarus, R. S., \& Folkman, S. (1984). Stress, Appraisal, and Coping. New York: Springer.

Martinent, G., \& Decret, J.-C. (2015). Motivational Profiles among Young Table-Tennis Players in Intensive Training Settings: A Latent Profile Transition Analysis. Journal of Applied Sport Psychology, 27, 268-287. https://doi.org/10.1080/10413200.2014.993485

Mellalieu, S. D., Hanton, S., \& Fletcher, D. (2006). A Competitive Anxiety Review: Recent Directions in Sport Psychology Research. In S. Hanton, \& S. D. Mellalieu (Eds.), Literature Reviews in Sport Psychology (pp. 1-45). New York: Nova Science.

Nicholls, A. R., \& Polman, R. C. J. (2007). Coping in Sport: A Systematic Review. Journal of Sports Sciences, 25, 11-31. https://doi.org/10.1080/02640410600630654

Nicolas, M., Gaudreau, P., \& Franche, V. (2011). Perception of Coaching Behaviors, Coping, and Achievement in a Sport Competition. Journal of Sport and Exercise Psychology, 33, 460-468. https://doi.org/10.1123/jsep.33.3.460

Ntoumanis, N., Biddle, S. J. H., \& Haddock, G. G. (1999). The Mediatory Role of Coping Strategies on the Relationship between Achievement Motivation and Affect in Sport. Anxiety, Stress, and Coping, 12, 299-327. https://doi.org/10.1080/10615809908250480

Roderick, M. (2006). The Work of Professional Football: A Labour of Love? Abingdon: Routledge.

Satorra, A., \& Bentler, E. M. (1994). Corrections to Test Statistics and Standard Enors in Covariance Structure Analysis. In A. von Eye, \& C. C. Clogg (Eds.), Latent Variables Analysis: Applications for Developmental Research (pp. 399-419). Thousand Oaks, CA: Sage.

Slimani, H. (2000). The Professionalization of French Football: A Model of Denial. Doctoral Thesis, Nantes: University of Nantes.

Smith, R. E., Smoll, F. L., \& Cumming, S. P. (2007). Effects of a Motivational Climate Intervention for Coaches on Young Athletes' Sport Performance Anxiety. Journal of Sport \& Exercise Psychology, 29, 39-59. https://doi.org/10.1123/jsep.29.1.39

Tabachnick, B. G., \& Fidell, L. S. (2007). Using Multivariate Statistics. Boston, MA: Pearson.

\section{Submit or recommend next manuscript to SCIRP and we will provide best service for you:}

Accepting pre-submission inquiries through Email, Facebook, LinkedIn, Twitter, etc. A wide selection of journals (inclusive of 9 subjects, more than 200 journals)

Providing 24-hour high-quality service

User-friendly online submission system

Fair and swift peer-review system

Efficient typesetting and proofreading procedure

Display of the result of downloads and visits, as well as the number of cited articles

Maximum dissemination of your research work

Submit your manuscript at: http://papersubmission.scirp.org/

Or contact ape@scirp.org 